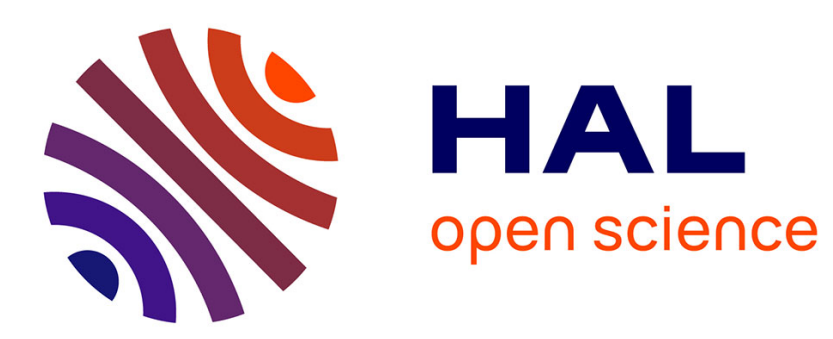

\title{
Practical Application of Matchmaking Problem: Trainee Allocation for Teachers
}

\author{
Maxime Morge, Eric Piette
}

\section{To cite this version:}

Maxime Morge, Eric Piette. Practical Application of Matchmaking Problem: Trainee Allocation for Teachers. International Conference on Practical Applications of Agents and Multi-Agent Systems (PAAMS), Yves Demazeau and Franco Zambonelli and Juan M. Corchado and Javier Bajo, Jun 2014, Salamanca, Spain. pp.195-206. hal-01058695

\section{HAL Id: hal-01058695 https://hal.inria.fr/hal-01058695}

Submitted on 27 Aug 2014

HAL is a multi-disciplinary open access archive for the deposit and dissemination of scientific research documents, whether they are published or not. The documents may come from teaching and research institutions in France or abroad, or from public or private research centers.
L'archive ouverte pluridisciplinaire HAL, est destinée au dépôt et à la diffusion de documents scientifiques de niveau recherche, publiés ou non, émanant des établissements d'enseignement et de recherche français ou étrangers, des laboratoires publics ou privés. 


\title{
Practical Application of Matchmaking Problem: Trainee Allocation for Teachers
}

\author{
Maxime Morge and Eric Piette \\ Laboratoire d'Informatique Fondamentale de Lille \\ Université Lille 1 \\ Cité Scientifique- F-59655 Villeneuve d'Ascq \\ maxime.morge@univ-lille1.fr, eric.piette@gmail.com
}

\begin{abstract}
In this paper, we tackle a complex real-world problem: trainee allocation for primary school teachers in a French teaching Academy. This complex real-world problem can be reduced into the wellknown Hospitals / Residents (HR) problem. However, the most difficult part consists of generating the preference lists according to the real constraints, priorities and wishes. Additionally, we adapt the $\mathbb{S}$ wing method to the HR problem and we apply it to this real-world problem in order to balance the different objectives. In this way, the $\mathbb{S}$ wing method decreases the management cost of the operation.
\end{abstract}

\section{Introduction}

Many real-world problems can be understood as matchmaking problems in which two sets need to be paired up: the assignment of junior physicians to hospitals [1], staff to faculties [2], students to colleges [3], children to schools [14], online matrimony [5], etc. Whatever the application domains are, the problem consists in finding the best matching between individuals. This problem was first studied in [6] which provides a constructive proof showing that every instance admits at least one admissible solution.

In this paper, we tackle a complex real-world problem: trainee allocation for primary school teachers in a French teaching Academy (in French, IUFM). For this purpose, the University Institute for Teachers Training has a program to allocate training practices according to the desiderata of trainees and the constraints of the supervisors. In order to be assigned, each teacher selects and orders two areas where she wants to be assigned. Additionally, priority is given to trainees having more children, then those working part-time and finally those with no car. Conversely, the diplomas of supervisors and the distances between the trainees and the supervisors allows to prioritize them. In order to manage the increasing number of recruitments, the allocation, which is performed manually, must be automated. It is worth noticing no optimization method can be applied due to scalability issues. This complex real-world problem can be reduced into the well-known Hospitals / Residents (HR) problem [6. However, the most difficult part consists of generating the preference lists according to

Y. Demazeau et al. (Eds.): PAAMS 2014, LNAI 8473, pp. 195-206 2014.

(C) Springer International Publishing Switzerland 2014 
the real constraints, priorities and wishes. Additionally, when we apply the existing algorithm for solving this problem we promote one community (e.g. the average distance) or another (e.g. the priority over trainees). Actually, even if the solution given by the Gale-Shapley algorithm is stable, it is the best one for one community but the worst for the other community [7]. By contrast, a recent method aims at reaching "fair" outcome: $\mathbb{S w i n g}$ [8]. In this paper, we adapt the $\mathbb{S} w i n g$ method to the HR problem and we apply it to a complex real-world problem in order to balance the different objectives. Since some constraints are relaxed, the Swing method decreases the management cost of the operation.

The paper is organized as follows. Section 2 introduces the background of our work. We adapt the $\mathbb{S}$ wing method in order to tackle this problem. Then, we present the real-world problem we address and we compare the solutions computed by the modified $\mathbb{S} w i n g$ method with some classical algorithms. Finally, section $[5$ discusses some related works and section $[6]$ concludes.

\section{Background}

The Hospitals / Residents (HR) problem was first defined in []. This problem is a many-one generalization of the well-known Stable Marriage Problem.

In the HR problem, each man corresponds to a resident and each woman corresponds to a hospital which can potentially be assigned to multiple residents up to some fixed capacity.

Definition 1 (HR). An instance of Hospitals / Residents problem of size $(n, m)$, with $n \geq 1$ and $m \geq 1$, is a couple $H R=\langle H, R\rangle$ with $|H|=n$ and $|R|=$ $m$ defined such that:

- $H=\left\{h_{1}, \ldots, h_{n}\right\}$ is a set of $n$ hospitals. Each hospital $h \in H$ has a positive integral capacity, denoted $c_{h}$, indicating the number of posts that $h$ has. Each hospital $h \in H$ has a preference list, denoted $\pi_{h}$, in which its ranks an acceptable set of residents in strict order;

- $R=\left\{r_{1}, \ldots, r_{m}\right\}$ is a set of $m$ residents, each resident $r \in R$ has a preference list, denoted $\pi_{r}$, in which she ranks an acceptable set of hospitals in strict order.

Given any individual $z \in H \cup R$, and given any potential partners $p_{1}, p_{2} \in H \cup R$, $z$ is said to prefer $p_{1}$ to $p_{2}$ if both $p_{1}$ and $p_{2}$ are in $\pi_{z}$ and $p_{1}$ precedes $p_{2}$ on $z$ 's preference list $\pi_{z}$.

A solution for an instance of HR is an assignment of residents in posts for each of the hospitals. The assignment of a resident is an hospital, possibly none (denoted $\theta$ ). The posts of an hospital are residents, possibly the empty set. Obviously, the assignment is mutual. Considering the assignment $M$, if $a_{M}\left(r_{k}\right)=$ $\theta, r_{k}$ is said to be unassigned, otherwise $r_{k}$ is assigned. Similarly, a hospital $h_{k} \in H$ is undersubscribed, full or oversubscribed if the corresponding number of residents in posts (denoted $\left|p_{M}\left(h_{k}\right)\right|$ ) is less than, equal to, or greater than $c_{k}$, respectively. In a matching, no resident is assigned to an unacceptable hospital, 
no hospital offers a post to an unacceptable resident, each resident is assigned to at most one hospital and no hospital is oversubscribed.

A matching for an instance of HR is stable there is no potential couple, called blocking pair, which threats the current matching. A blocking pair prefers to be assigned together rather than according to the current matching. Considering a matching $M$ for a HR problem, a couple $(h, r)$ is blocking if $r$ prefers $h$ to her current assignment and: either $h$ is undersubscribed; or $h$ prefers $r$ to at least one of its posts. A stable matching is called an admissible solution.

[6] provides a constructive proof showing that every instance of HR admits at least one admissible solution: an algorithm called the resident-oriented GaleShapley algorithm (RGS for short). In RGS, each unassigned resident, which is not desperate (alone and with a non-empty preference list), sends a proposal to her preferred hospital which accepts. If this latter is oversubscribed, then the hospital fires the worst resident having a post in this hospital. If the hospital is full, it deletes from its preference list all the residents who are worst than the residents having a post and reciprocally.

A counterpart of the RGS algorithm, known as the hospital-oriented GaleShapley algorithm (HGS for short), involves hospitals offering posts to residents. In HGS, each hospital which is not desperate (undersubscribed and with at least one resident in its preference list which is not assigned to it) proposes a post to the best one who accepts. If the resident was already assigned to a different hospital, then the resident is first unassigned. Then, the resident deletes from her preference list all the hospitals which are worst than the current hospital and reciprocally.

The RGS algorithm terminates with the resident-optimal stable matching (denoted $M_{r}$ ) in which each assigned resident has the best hospital that she could achieve in any stable matching and each unassigned resident is unassigned in every stable matching. $M_{r}$ is worst-possible for the hospitals : if $M$ is any other stable matching, then every hospital $h \in H$ prefers each resident in $p_{M}(h)$ to each resident in $p_{M_{r}}(h) \backslash p_{M}(h)$. The HGS terminates with the hospital-optimal stable matching (denoted $M_{h}$ ) in which every full hospital $h$ is assigned with its $c_{h}$ best partners and every undersubscribed hospital is assigned to the same set of residents in every stable matching. In $M_{h}$, each assigned resident has the worst hospital that she could achieve in any stable matching and each unassigned resident is unassigned in every stable matching. In general, there may be other stable matchings which cannot be reached by these two algorithms.

\section{Swing}

We adapt here the method $\mathbb{S}$ wing which was initially proposed in 8 to solve Stable Marriage Problem in order to tackle HR. Swing may reach some stable matchings which are not the output of RGS and HGS.

In the RGS and HGS algorithms, each community is given a role (proposer or responder). In the $\mathbb{S}$ wing method, the agents (residents or hospitals) alternatively play both of them in many bilateral negotiations from which the solution 
emerges. Swing realizes the minimal concession strategy [9] to reach a stable matching in order to be more equitable than the outputs of RGS and HGS. Based on this strategy, an agent goes first to its preferred partner. If that fails, the agent concedes, which consists in the withdrawal of its expectation, and so it sends a proposal to the following partners in its preference list. Meanwhile, the potential partners play the role of responder: these agents receive some proposals they can accept or reject depending on their concession levels. When all the agents are fully assigned or desperate, the $\mathbb{S}$ wing method stops.

The Swing method adopts an agent-based methodology for solving an economics problem of matchmaking since it focus on the interaction between the agents and the link between their satisfaction and the market. In the Swing method, each hospital and each resident is represented by an agent $a \in H \cup R$. At each step, the agent $a$ is represented by a concession level $\left(\kappa_{a} \in\left[0,\left|\pi_{a}\right|\right]\right)$ and its assignment status $\left(\sigma_{a} \in\{\top, \perp\}\right)$. We note $\pi_{z}[1]$ the most preferred partner of $z, \pi_{z}[2]$ the second most preferred partner of $z$, and so on. If $\pi_{z}[k]=\lambda$, then $\operatorname{regret}_{z}(\lambda)=k$. We define the concession level as the maximum rank in the preference list that the agent considers as acceptable at a certain time. $\kappa_{z}=1$ means that the agent focus on its most preferred partner and so the other potential partners are not acceptable. A resident $r$ is fully assigned $\left(\sigma_{r}=\top\right)$ if she is assigned $\left(a_{M}(r) \neq \theta\right)$. A hospital $h$ is fully assigned $\left(\sigma_{r}=\top\right)$ if it is full $\left(\left|p_{M}(h)\right|=c_{h}\right)$. Initially, $\sigma_{a}=\perp$ and $\kappa_{a}=1$ for all the agents. The preference lists $\pi_{a}$ are different from one agent to another.

In $\mathbb{S}$ wing, hospitals propose and residents respond alternatively (cf Algo. II). In the odd steps, the hospitals play the role of proposers and the residents play the role of responders. In the even steps, the roles are swapped. Each proposer sends a proposal to the acceptable partners from the preferred ones to the least preferred ones. As soon as a responder accepts this proposal:

1. the proposer and the responder may divorce;

2. the proposer and responder are assigned;

3. the concession level of the proposer/responder are moved such that, if they are fully assigned, then they will only accept better partners.

It is worth noticing that, at each step, a proposer stops to send proposals as soon as it is fully assigned. If all the responders reject its proposals, the proposer will concede. When all the agents are fully assigned or desperate, the Swing method stops. An agent is desperate if this is an hospital (resp. a resident) which is undersubscribed (resp. unassigned) and it has reached the maximal concession level (the preference list has been fully explored).

At each step, the proposer and the responder may divorce (cf Algo. 21). If a member of the new couple is fully assigned, its previous (or one of its previous) partner is unassigned and so this latter may need to concede.

\section{Practical Application}

In this section, we present the real-world problem we tackle: the trainee allocation for primary school teachers in a French teaching Academy. For this purpose, the 


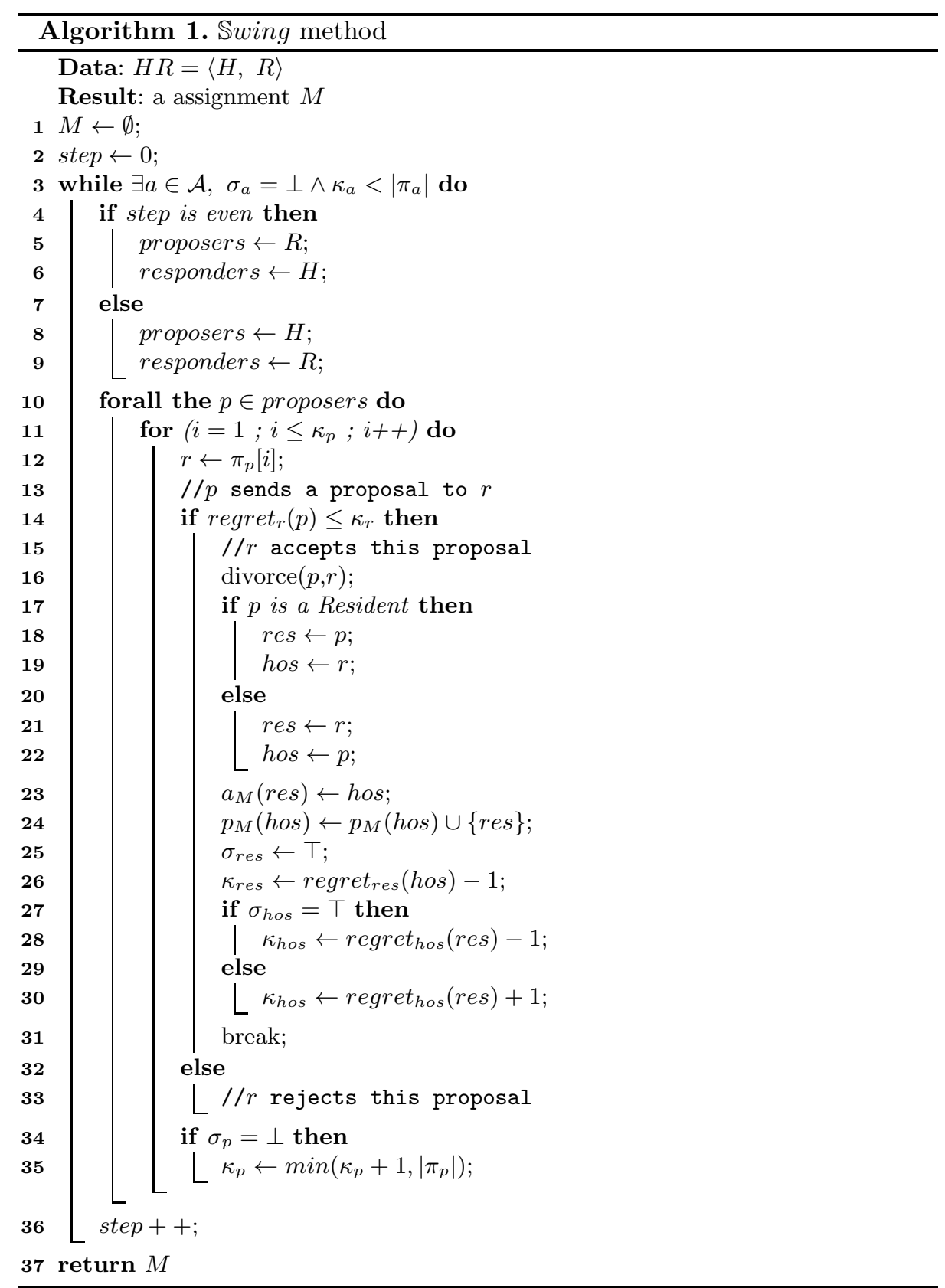




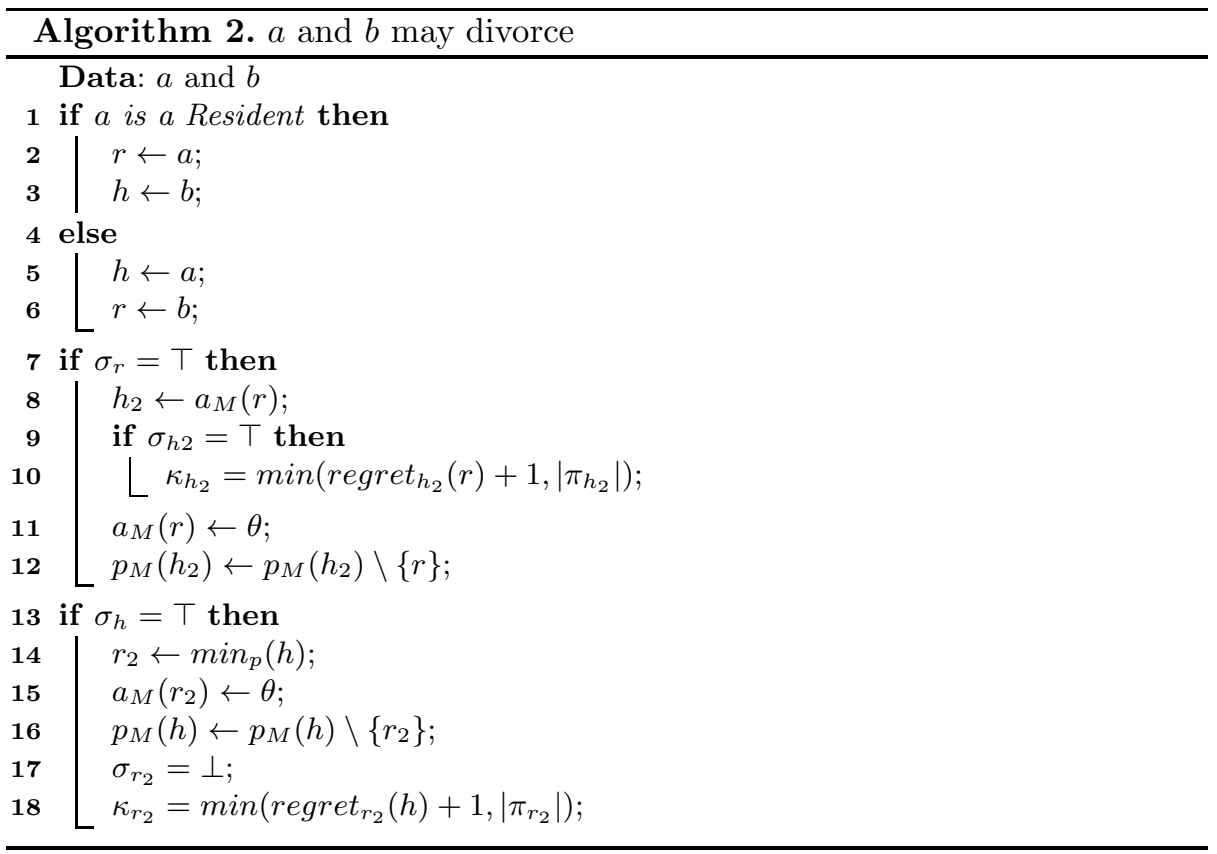

University Institute for Teachers Training (in French IUFM) has a program to allocate training practices according to the desiderata of trainees and the constraints of the supervisors. We show here how this real-world problem is modeled and can be computed with the HR solving methods.

\subsection{Overview}

Each teacher must complete 3 internships, one per each quarter. Conversely, each supervisor can manage at most 2 trainees for each quarter. The region is divided into some areas and subareas. Each subarea is composed of cities where schools are located. In order to be assigned, each teacher expresses two wishes, i.e she selects and orders two areas where she wants to be assigned.

The problem is represented in Fig. 11 with an ER-model. The supervisors teach in different classrooms. Each classroom is included in one or more levels. The French primary education is divided into three levels:

- level L1 includes 3 classrooms called TPS, PS and MS;

- level L2 includes 3 classrooms called GS, CP and CE1;

- level L3 includes 3 classrooms called CE2, CM1 and CM2.

When a supervisors teaches in a classroom which is included in a level, we consider she teaches at this level. However, if she simultaneously teaches in GS and MS (or PS), then we consider she also teaches at level L1. 


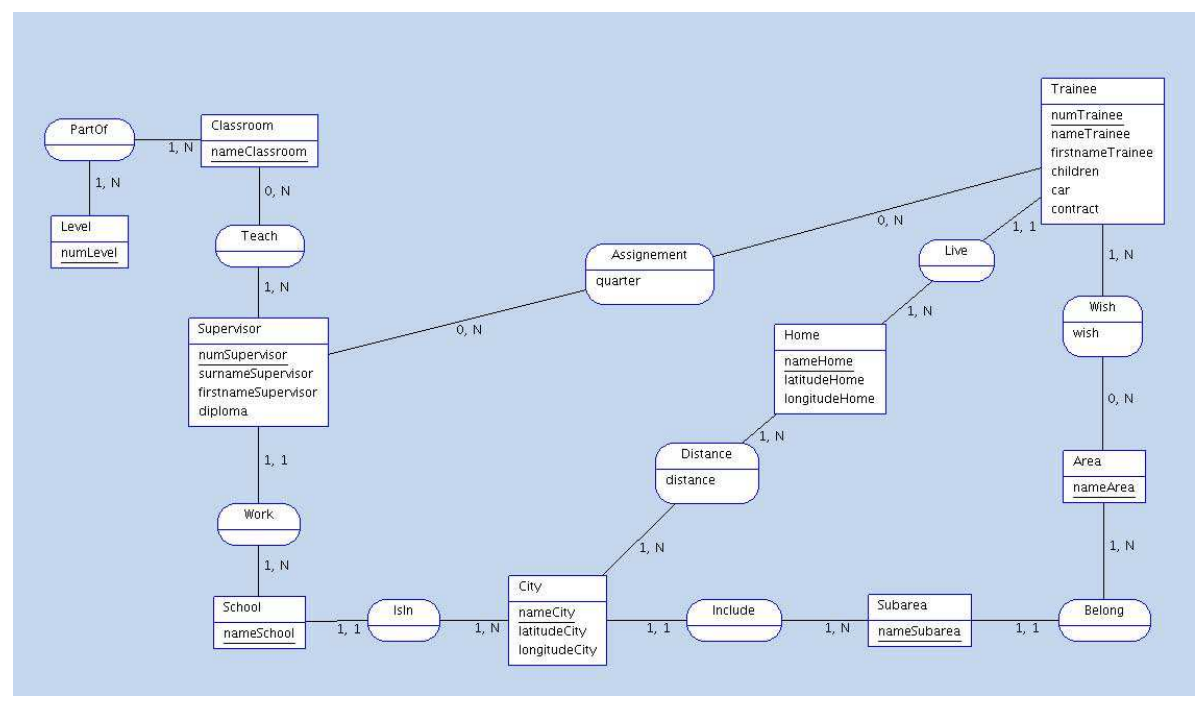

Fig. 1. ER model representing the inputs and the output of the problem

The IUFM aims at assigning an internship per quarter for each trainee respecting her wishes. Moreover, the IUFM wants to minimize the refunding due to the travels. That is the reason why we add the relation "Distance" between the homes of trainees and the cities where schools are. The distinction between these two entities allows to decrease the number of distances which are computed.

Priority is given to trainees having more children, then those working parttime and finally those with no car. Conversely, the diplomas of supervisors (EMF, CAFIPEMF or MAT) allows to prioritize them. Finally, the IUFM aims at minimizing the number of employed supervisors.

\subsection{Mapping}

An instance of Primary School Teachers problem (PST) is a couple PST = $\langle S, T\rangle$ where $S$ is a set of supervisors and $T$ is a set of trainees. An instance of PST can be reduced into 3 instances of HR, each of them corresponding to a quarter. In order to solve an instance of PST, we generate the corresponding instances of HR, solve them and translate the solutions.

Let us consider an instance of PST. In order to reach the assignment $W_{i}$ for the quarter $i$, we transform PST into an instance $\mathrm{HR}_{i}$ in order to compute a matching with RGS, HGS or Swing. This matching $M_{i}$ allows to deduce $W_{i}$. Informally, the transformation is performed using Tab. [1

The mapping between the agents for an instance PST and the agents for an instance of HR is performed by a bijection. For each quarter, the corresponding instance of $\mathrm{HR}$ is generated and resolved. Therefore, $M_{i}$ is translated into $W_{i}$.

The transformation of an instance of PST into an instance of HR is performed in 3 steps. Firstly, supervisors are mapped to hospitals and trainees are 
Table 1. Mapping between HR and PST

\begin{tabular}{l|l} 
PST & HR \\
\hline Supervisor & Hospital \\
Trainee & Resident \\
Constraints, priorities, and wishes & Preference list
\end{tabular}

mapped to residents. Secondly, the preference lists of residents and hospitals are generated. Finally, the instance of HR is returned.

The most difficult part consists in generating the preference lists of HR according to the real constraints, priorities, and wishes within PST expressed by the trainees and supervisors. The preference lists of residents are generated as follows. Firstly, the preference list of a resident is set up with the hospitals which map with the supervisors in accordance with the wishes of the corresponding trainee. Secondly, these hospitals are divided in accordance with the levels of the corresponding supervisors in order to give priority to the levels which are not performed by the corresponding trainee. Finally, the hospitals corresponding to supervisors who teach in classrooms already practiced by the corresponding trainee are deleted. The generation of the preference lists for the hospitals is performed as follows. For each hospital, the preference list is set up with the residents who consider it. Residents are sorted according to the number of children of the corresponding trainee, then according to the working time, and finally according to the car.

\subsection{Experiments}

We consider here data for the 2012 trainee allocation. 356 junior teachers must find 3 training practices supervised by one of the 783 senior teachers. As described previously, we transform this instance of PST into 3 instances of HR. Then, we resolve them using RGS, HGS or Swing (see Algo. (I). It is worth noticing no optimization method have been applied such as SMP2 [10] or DisFC [11] due to scalability issues. The experiments have been performed by a MacBookPro (2.6 GHz Intel Core i7 with 8 Go RAM). Each method reaches a solution in around twenty minutes. $80 \%$ of the computational time is consumed by the generation of the preference lists which are different for each quarter since: i) the supervisors must be sorted according to the wishes, the diplomas, the distance and the levels which are not performed; ii) the trainees must be sorted according to the number of children of the corresponding trainee, then according to the working time, and finally according to the car. The assignments are denoted $W_{R G S}, W_{H G S}$ and $W_{\text {Swing }}$.

Table 1 compares the outcomes of the three methods. We can note that all the trainees are allocated according to these methods. Indeed, the number of available supervisors is greater than the number of trainees. Contrary to HGS, the RGS algorithm - which promotes the trainees - and the $\mathbb{S}$ wing method, allow (almost all trainees) to perform a training practice at each level. Whatever the 
method used, all the matchings are compliant with the wishes expressed by the trainees. Even if the HGS algorithm does not give priority to trainees, HGS as the RGS algorithm give the priority to the first wishes of trainees. Its seems that this soft constraint is relaxed by the $\mathbb{S}$ wing method. The average distance between the home of a candidate and the place of the training practice is lower for $W_{R G S}$ (promoting trainees) than $W_{H G S}$. The average distance for $W_{\mathbb{S} w i n g}$ is in between. Since some constraints are relaxed, the $\mathbb{S} w i n g$ method allows to decrease the cost of management (by reducing the number of employed supervisors) much more than HGS and RGS.

Table 2. Overview (at top) and distribution of trainees/diplomas (at bottom)

\begin{tabular}{|c|c|c|c|c|}
\hline \multicolumn{2}{|l|}{ Criteria } & $W_{R G S} \mid$ & $W_{H G S}$ & $W_{\text {Swing }}$ \\
\hline \multicolumn{2}{|c|}{ Trainees with 3 practices } & 356 & 356 & 356 \\
\hline \multicolumn{2}{|c|}{ Trainees with 3 levels } & 348 & 266 & 356 \\
\hline \multicolumn{2}{|c|}{ Trainees with wish \# 1} & 185 & 190 & 65 \\
\hline \multicolumn{2}{|c|}{ Average distance $(\mathrm{km})$} & 22.26 & 29.29 & 23.58 \\
\hline \multicolumn{2}{|l|}{$\mathrm{Nb}$ of supervisors } & 496 & 348 & 64 \\
\hline Diploma & $W_{R G S}$ & $W_{H G S}$ & $W_{\mathbb{S} w}$ & \\
\hline$\overline{\mathrm{EMF}}$ & $41.23 \%$ & $32.02 \%$ & \begin{tabular}{l|l}
7 & 15.78
\end{tabular} & \\
\hline CAFIPEMF & $39.13 \%$ & $27.05 \%$ & 6.89 & \\
\hline MAT & $18.76 \%$ & $21.16 \%$ & $\%$ & \\
\hline
\end{tabular}

Table 1 shows the distribution of supervisors over diplomas. For instances, $41.23 \%$ of supervisors having the best diploma (EMF) are required by the RGS algorithm. The RGS and HGS algorithms employ the supervisors with the best diplomas as much as possible. The RGS algorithm involves more specifically the supervisors with the best diplomas since the preference list of the trainees are generated depending on these diplomas. Since the $\mathbb{S}$ wing method involves only 64 supervisors (12 with EMF, 44 with CAFIPEMF and 7 with MAT), it is hard to say if the matching takes into account the diplomas of the supervisors.

As said previously, priority is given to trainees having more children, those working part-time and finally those who have no car. Table 3 summarizes for each of these categories, the number of trainees and the average distance. In 2012, few trainees having children are involved. That is the reason why we cannot check that the average distance decreases when the number of children increases. If we consider only trainees with no child, it seems that the trainees working part-time are promoted. Since the trainees with no car represent two-thirds of the cohort, the corresponding rule cannot be applied whatever the algorithm is.

Table. 4 summarizes and compares the outcomes of our three methods. ++, + , and -, means that a criteria is completely, moderately and badly fullfilled respectively. According to our experiments, no method seems to satisfy all the criteria. While RGS seems to give priority to trainees (level coverage, average distance, diploma), HGS is adapted to give priority to the supervisors (i.e. children, part-time, car). The $\mathbb{S} w i n g$ method balances both aspects. Actually, this 
Table 3. Average distances for trainees

\begin{tabular}{|c|c|c|c|c|c|c|}
\hline $\begin{array}{l}\text { Nb of } \\
\text { child. }\end{array}$ & $\mid \begin{array}{r}\text { Work. } \\
\text { time }\end{array}$ & Car & $\begin{array}{r}\mathrm{Nb} \text { of } \\
\text { trainee }\end{array}$ & $W_{R G S}$ & $\left|W_{H G S}\right|$ & $W_{\text {swing }}$ \\
\hline \multirow{4}{*}{$4]$} & \multirow[t]{2}{*}{ Part } & $\mathrm{No}$ & 0 & - & - & - \\
\hline & & Yes & 0 & - & -1 & - \\
\hline & \multirow[t]{2}{*}{ Full } & $\mathrm{No}$ & 1 & 2.01 & 20.20 & 2.01 \\
\hline & & \begin{tabular}{|l|} 
Yes \\
\end{tabular} & 0 & - & - & - \\
\hline \multirow{4}{*}{3} & \multirow[t]{2}{*}{ Part } & $\mathrm{No}$ & 0 & - & - & - \\
\hline & & Yes & 0 & - & - & - \\
\hline & \multirow[t]{2}{*}{ Full } & $\mathrm{No}$ & 2 & 5.84 & 67.90 & 6.78 \\
\hline & & Yes & 0 & - & - & - \\
\hline \multirow{4}{*}{2} & \multirow[t]{2}{*}{ TPS } & $\mathrm{No}$ & 0 & - & - & - \\
\hline & & Yes & 0 & - & - & - \\
\hline & \multirow[t]{2}{*}{ Full } & No & 1 & 45.79 & 45.80 & 45.79 \\
\hline & & Yes & 5 & 10.79 & 16.30 & 11.45 \\
\hline \multirow{4}{*}{1} & \multirow[t]{2}{*}{ TPS } & No & 0 & - & - & - \\
\hline & & Yes & 1 & 6.92 & 73.90 & 7.38 \\
\hline & \multirow[t]{2}{*}{ Full } & $\mathrm{No}$ & 12 & 17.03 & 20.20 & 17.35 \\
\hline & & Yes & $\overline{2}$ & 6.34 & 81.90 & 8.18 \\
\hline \multirow{4}{*}{0} & \multirow[t]{2}{*}{ TPS } & No & 13 & 7.58 & 15.00 & 7.94 \\
\hline & & Yes & $\overline{9}$ & $\overline{19.39}$ & 16.30 & 19.92 \\
\hline & \multirow[t]{2}{*}{ Full } & \begin{tabular}{|l|}
$\mathrm{No}$ \\
\end{tabular} & 202 & 27.43 & 35.70 & 29.01 \\
\hline & & Yes & 108 & 20.42 & 22.60 & 17.68 \\
\hline
\end{tabular}

method seems to relax the constraints related to the wishes. Since these constraints have been relaxed, the Swing method allows to increase the coverage of levels and to decrease the number of supervisors who are employed and so, the management cost of the matching.

\section{$5 \quad$ Related Works}

The most famous real-world application for matchmaking problems is the National Resident Matching Program (NRMP) which manage the entry-level labor market for new physicians in the United States [1]. Each year, approximately 20,000 jobs are filled. In 2012, 38,777 aspiring medical residents applied for 26,772 available resident positions. For this purpose, each applicant submits a rank order list of positions for which she has interviewed and each residency program submit a rank order list of applicants they have interviewed and the number of positions to fill. Even if the number of participants for this application is greater than in our experiments, it is a straightforward application of the HR problem while solving a PST problem requires the generation of complex preference lists.

College admissions in China are centralized processes via standardized tests 3 . Therefore, the same preferences list is generated according to the test scores for all the schools. The Gale-Shapley algorithm is used for public school admissions in Boston and New-York [1]. Since, the inputs include the preferences of pupils over school and the priority levels of pupils (a pupil has priority to attend the 
Table 4. Comparison of the methods for solving HR applied to an instance of PST

\begin{tabular}{l|c|c|c} 
Criteria & RGS & HGS & Swing \\
\hline 3 training practices & ++ & ++ & ++ \\
3 levels & + & - & ++ \\
Wishes & + & + & - \\
\hline Distance & ++ & - & + \\
Nb. of supervisors & - & + & ++ \\
\hline Priority to diplomas & ++ & + & $?$ \\
\hline Priority to children & $?$ & $?$ & $?$ \\
Priority to part-time & + & + & + \\
Priority to "no car" & - & - & -
\end{tabular}

same school as an older sibling, pupils who are living in the school's walk zone have priority), the generation of the preference lists for schools is quite simple. In the daycare system in Denmark 4, priorities are imposed by local municipality (e.g. all schools give priority to their currently enrolled children and to the children with special needs). Therefore, the generation of priority is also straightforward.

In [5], the application domain is online matrimony in India. They propose to generate the preference list of participants according to the characteristics of the potential partners using fuzzy analytical hierarchy process considering multiple criteria. This method performs pairwise comparison of candidates attribute-wise since both women and men value physical attributes, such as age and weight, and those choices are assortative along age, height and education. Even if this application requires the generation of complex preference lists, the resulting matching is not evaluated.

\section{Conclusion}

In this paper, we have addressed a complex real-world problem: trainee allocation for primary school teachers in a French teaching Academy. Since we have presented the inputs and the outputs of such problem called Primary Schools Teachers (for short, PST), we have shown that an instance of PST can be reduced into 3 instances of the well-known Hospitals / Residents (HR) problem. However, this transformation requires the generation of complex preference lists. The preference list of each resident takes into account the wishes of the corresponding trainees, the diplomas of the supervisors, the distance between them, etc. The preference list of each hospital takes into account the priority over trainees which depends on the number of children, if they are part-time and if they have cars. In order to solve the corresponding instances of HR, we have tried the classical algorithms (the resident-oriented Gale-Shapley algorithm and the hospital-oriented Gale-Shapley) and we have also adapted a "fair" method called $\mathbb{S}$ wing. Our first experiments seem to be in conformance with the fact that this latter balances the criteria which promote the trainees (e.g. average distance) and the criteria which promote the supervisor (i.e the priority over 
the trainees). Since some constraints are relaxed, the $\mathbb{S}$ wing method decreases the number of supervisors who are employed and so the management cost of the operation. However, it would be interesting to confront our first conclusion with the data of future campaigns.

\section{References}

1. Roth, A., Peranson, E.: The redesign of the matching market for american physicians: Some engineering aspects of economic design. AER 89(4), 748-780 (1999)

2. Baiou, M., Balinski, M.: Admissions and recruitment. AMM 110(5), 386-399 (2003)

3. Zhang, H.: Analysis of the Chinese college admission system. PhD thesis, University of Edinburgh (2009)

4. Kennes, J., Monte, D., Tumennasan, N.: The daycare assignment problem. Technical report, Department if Economics and Business, Aarhus University (2011)

5. Joshi, K., Kumar, S.: Matchmaking using fuzzy analytical hierarchy process, compatibility measure and stable matching for online matrimony in india. Journal of MCDA 19, 57-66 (2012)

6. Gale, D., Shapley, L.S.: College admissions and the stability of marriage. AMM 69, 9-14 (1962)

7. Knuth, D.: Mariage stables. Les Presses de l'Université de Montréal (1971)

8. Everaere, P., Picard, G., Morge, M.: Minimal concession strategy for reaching fair, optimal and stable marriages (extended abstract). In: Proc. of AAMAS, pp. 1319-1320 (2013)

9. Rosenschein, J., Zlotkin, G.: Rules of encounter: designing conventions for automated negotiation among Computers. MIT press (1994)

10. Gelain, M., Pini, M., Rossi, F., Venable, K., Walsh, T.: Local search algorithms on the stable marriage problem: Experimental studies. In: Proc. of ECAI, pp. 1085-1086 (2010)

11. Brito, I., Meseguer, P.: Distributed stable matching problems. In: van Beek, P. (ed.) CP 2005. LNCS, vol. 3709, pp. 152-166. Springer, Heidelberg (2005) 